\title{
Can magnifying endoscopy with narrow-band imaging discriminate between carcinomas and low grade adenomas in gastric superficial elevated lesions?
}

Authors

Institutions
Takashi Nonaka', Masahiko Inamori², Yasushi Honda', Kenji Kanoshima', Yumi Inoh' ${ }^{1}$, Mizue Matsuura', Shiori Uchiyama ${ }^{1}$, Eiji Sakai', Takuma Higurashi ${ }^{1}$, Hidenori Ohkubo ${ }^{1}$, Hiroshi lida ${ }^{1}$, Hiroki Endo ${ }^{1}$, Koji Fujita ${ }^{1}$, Akihiko Kusakabe ${ }^{2}$, Kazuhiro Atsukawa ${ }^{3}$, Hisao Takahashi ${ }^{3}$, Yoko Tateishi ${ }^{4}$, Shin Maeda², Kenichi Ohashi ${ }^{4}$, Atsushi Nakajima'

Institutions are listed at the end of article. submitted 30. May 2016 accepted after revision 9. September 2016

\section{Bibliography}

Dol http://dx.doi.org/ 10.1055/s-0042-117632 Published online: 20.10.2016 Endoscopy International Open 2016; 04: E1203-E1210

(c) Georg Thieme Verlag KG Stuttgart · New York E-ISSN 2196-9736

\section{Corresponding author}

\section{Masahiko Inamori, MD PhD}

Department of Medical

Education

Yokohama City University

School of Medicine

3-9 Fukuura

Kanazawa-ku

Yokohama

236-0004

Japan

Fax: +81-45-7843546

inamorim@med.yokohama-cu. ac.jp
Background and study aims: The aim of this study was to investigate the capability of magnifying endoscopy with narrow-band imaging (ME-NBI) to discriminate between early carcinomas (EC) and low grade adenomas (LGA) in gastric superficial elevated epithelial neoplasias.

Patients and methods: We investigated 100 consecutive cases of gastric superficial elevated epithelial neoplasias that were removed using endoscopic submucosal dissection. The pathological diagnostic criteria were based on the revised Vienna classification; category 4 (mucosal high grade neoplasia) and category 5 (submucosal invasion by carcinoma) lesions were diagnosed as EC, whereas category 3 (mucosal low grade neoplasia) lesions were diagnosed as LGA. The associations between the postoperative pathological diagnoses and the ME-NBI findings were analyzed, and included the shape, specification, and area of irregularity in the microvascular architecture (MV) and the microsurface structure (MS).

Results: Seventy-nine EC and 21 LGA cases diag-

\section{Introduction}

$\nabla$

Superficial elevated epithelial neoplasias of the stomach can be broadly divided into early carcinomas (EC) and low grade adenomas (LGA). Early detection and curative treatment are the best strategies for gastric carcinomas, and the indications for endoscopic treatment for EC have been established through the introduction of endoscopic submucosal dissection (ESD) in Japan [1, 2]. However, therapeutic strategies for LGA, which is defined as a benign tumor, currently vary among facilities $[3,4]$. Additionally, in contrast to gastric depressed epithelial neoplasias, which generally consist of carcinomas, the endoscopic discrimination of EC and LGA in superficial elevated epithelial neoplasias is difficult in the clinical setting. Although a biopsy method is considered an essential modality for making a differential di- nosed postoperatively were evaluated retrospectively. The lesion size (median; range $(\mathrm{mm})$ ) was significantly larger in the EC group $(14 ; 2-95)$ compared to the LGA group $(5 ; 2-16)(P<0.001)$. Wavy forms in the MV shapes $(P=0.031)$, extension in the MV specifications $(P=0.035)$, and area with MV irregularity ( $P=0.001$ ) were found to be statistically significant predictive findings for EC. Villous forms in the MS shapes $(P=0.026)$, enlargement in the MS specifications $(P=0.044)$, and area with MS irregularity $(P=0.021)$ were also found to be statistically significant predictive findings for EC. The rates of preoperative sensitivity, specificity, and diagnostic accuracy of ME-NBI for discriminating EC were $86.1 \%, 38.9 \%$, and $75 \%$, respectively.

Conclusions: The present study suggests that MENBI is useful for the differential diagnosis of EC and LGA in gastric superficial elevated epithelial neoplasias.

\section{Study registration: UMIN000012925.}

agnosis, we have often experienced that lesions diagnosed as LGA using preoperative biopsies were actually EC after removal by ESD. Indeed, several studies have indicated that preoperative biopsy sampling is inadequate for a correct diagnosis $[5,6]$. Therefore, it is important for endoscopists to identify optical signs that can be used to discriminate EC and LGA without being solely dependent upon biopsy results.

Recently, magnifying endoscopy with narrowband imaging (ME-NBI) has been reported as a useful modality for the accurate diagnosis of epithelial neoplasias in the stomach [7-9]. Although several published reports have focused on the differential diagnosis between EC and LGA using ME-NBI [10-13], some of these studies have discriminated actual EC from LGA diagnosed using a preoperative biopsy and other studies have been conducted without distinction of the macroscopic 
Table 1 Revised Vienna classification of gastrointestinal epithelial neoplasias.

\begin{tabular}{|l|l|}
\hline Category & Diagnosis \\
\hline 1 & Negative for neoplasia \\
\hline 2 & Indefinite for neoplasia \\
\hline 3 & $\begin{array}{c}\text { Mucosal low grade neoplasia } \\
\text { Low grade adenoma/dysplasia }\end{array}$ \\
\hline 4 & Mucosal high grade neoplasia \\
\hline 4.1 & High grade adenoma/dysplasia \\
\hline 4.2 & Non-invasive carcinoma (carcinoma in situ) \\
\hline 4.3 & Suspicious for invasive carcinoma \\
\hline 4.4 & Intramucosal carcinoma \\
\hline 5 & Submucosal invasion by carcinoma \\
\hline
\end{tabular}

appearance of the lesions as elevated or depressed lesions. Namely, the characteristics of ME-NBI findings for EC with a superficial elevated gross appearance and the differential diagnosis between EC and LGA using ME-NBI have not yet been sufficiently investigated. The present study was conducted to investigate whether ME-NBI was useful for the differential diagnosis of EC and LGA in gastric superficial elevated epithelial neoplasias.

\section{Methods}

$\nabla$

\section{Subjects and materials}

We examined a total of 100 superficial elevated gastric epithelial neoplasias, regardless of whether the preoperative diagnosis was EC or LGA by biopsy, in 91 consecutive patients who underwent ME-NBI followed by ESD during the period from October 2009 through March 2014 at the Yokohama City University Hospital. Superficial elevated lesions were defined as having a gross appearance with less than $3 \mathrm{~mm}$ elevation based on the Japanese classification of gastric carcinoma [1]. With regard to gross appearance, protrusions with $3 \mathrm{~mm}$ or greater elevation, lesions that were completely flat, depressed or ulcerated without any superficial elevated components, and predominantly depressed lesions with non-neoplastic elevated borders or a central elevation were not included. Additionally, local recurrent lesions and cases with a past history of surgical resection of the stomach were excluded even if their endoscopic appearances satisfied the criteria in terms of gross appearance.

\section{Indications for ESD at our institution}

The indications for ESD in cases of gastric epithelial neoplasias diagnosed as EC by preoperative biopsies were in accordance with the recommendations of Gotoda et al. [14]. All cases of gastric superficial elevated epithelial neoplasias diagnosed as LGA by preoperative biopsies were recommended to undergo ESD with sufficient informed consent. However, whether endoscopic resection or follow-up would be chosen was eventually left to the discretion of each patient.

\section{Pathological investigation}

All resected neoplasias were fixed in $10 \%$ buffered formalin and segmented at 2-mm intervals. Each section was stained with hematoxylin and eosin and evaluated by two pathologists at our institution. The diagnoses were based on the revised Vienna classification ( Table 1) [15]. For the purpose of this study, we recategorized the revised Vienna classification category 4 (mucosal high grade neoplasia) and category 5 (submucosal invasion by carcinoma) as EC, and category 3 (mucosal low grade neoplasia) as LGA.

\section{Data analysis}

The clinical and endoscopic characteristics were retrospectively reviewed for all patients using the proprietary database. The endoscopic image quality was evaluated by the three observers who were familiar with ME-NBI and accredited by the Japan Gastroenterological Endoscopy Society, and no patient was excluded from the present study because of poor endoscopic records. Additionally, the endoscopic findings were determined by consensus among the aforementioned three observers who were blinded to any preoperative or postoperative histological results.

We assessed the clinical and endoscopic characteristics, which included age and sex, lesion location, lesion maximal diameter $(\mathrm{mm})$, lesion color, and the following ME-NBI findings: the shape (closed loop, open loop, kinked, linear, wavy, branched, coiled, and dot) ( $\bullet$ Fig. 1), specifications (dilatation, narrowness, extension and caliber change) ( $\bullet$ Fig. 2), and area of irregularity of the microvascular architecture (MV) ( $\bullet$ Fig.3), and the shape (circle, circles in villi, tubular, curved, oval, villous, polygonal, and amorphous) ( Fig.4), specifications (enlargement, miniature, and extension) ( $\bullet$ Fig.5), and area of irregularity of the microsurface structure (MS) ( $\bullet$ Fig. 6). The definitions of the shape and specification of the MV/MS were as follows. Regarding the shape of MV -closed loop: a loop shape with closed circuit like a ring; open loop: a loop shape without closed circuit like the character "C"; linear: a straight shape and kinked: a straight shape with sharp bends. Regarding the shape of MS-circle: a small round shape which surrounds a round pit; circles in villi: accumulation of circular microsurface structures in villus-like component; curved: a crescent shape; and amorphous: disappearance of microsurface structures. The specifications of the MV/MS were judged compared with the background non-neoplastic mucosa. Regarding the specification of MV-dilatation: the presence of microvessels whose caliber is more than twice as thick as the caliber of the non-neoplastic microvessels; narrowness: the presence of microvessels whose caliber is less than half as thin as the caliber of the non-neoplastic microvessels; extension: the presence of microvessels whose length is more than twice as long as the length of the non-neoplastic microvessels; and caliber change: the presence of microvessels whose caliber becomes partially less than half or more than twice the size of the main size. Regarding the specification of MS - enlargement: the presence of MS whose width is more than twice as large as the width of the non-neoplastic MS; miniature: the presence of MS whose width is less than half as small as the width of the non-neoplastic MS; and extension: the presence of MS whose length is more than twice as long as the length of the non-neoplastic MS. The area of MV/MS irregularity was defined as having one of the following: non-uniformity, irregular arrangement or asymmetric distribution in middle-magnification images obtained using ME-NBI. The localized site was classified as the upper (U), middle (M) and lower $(\mathrm{L})$ part by lines connecting the trisected points on the lesser and greater curvatures according to the Japanese classification of gastric carcinoma [1]. The lesion's maximal diameter was determined by measuring the resected specimen. Moreover, we also investigated the lesion size, which was divided into two groups: greater than or equal to $20 \mathrm{~mm}$ in diameter or less than $20 \mathrm{~mm}$ in diameter. The color of the lesion was classified as reddish or whitish compared with the surrounding non-neoplastic mucosa. 

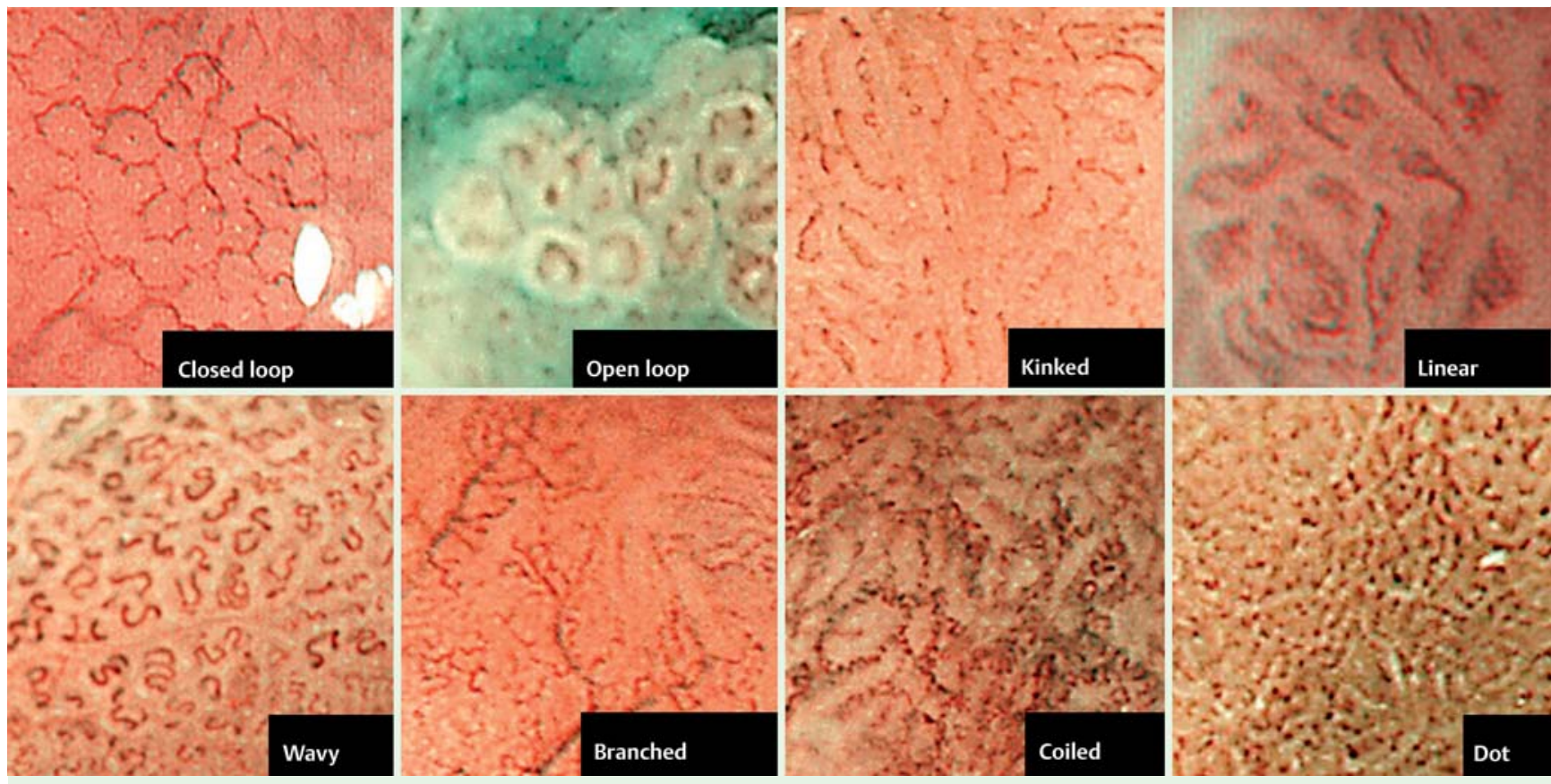

Fig. 1 Eight patterns of the microvascular architecture (MV) shape using magnifying endoscopy with narrow-band imaging (ME-NBI).
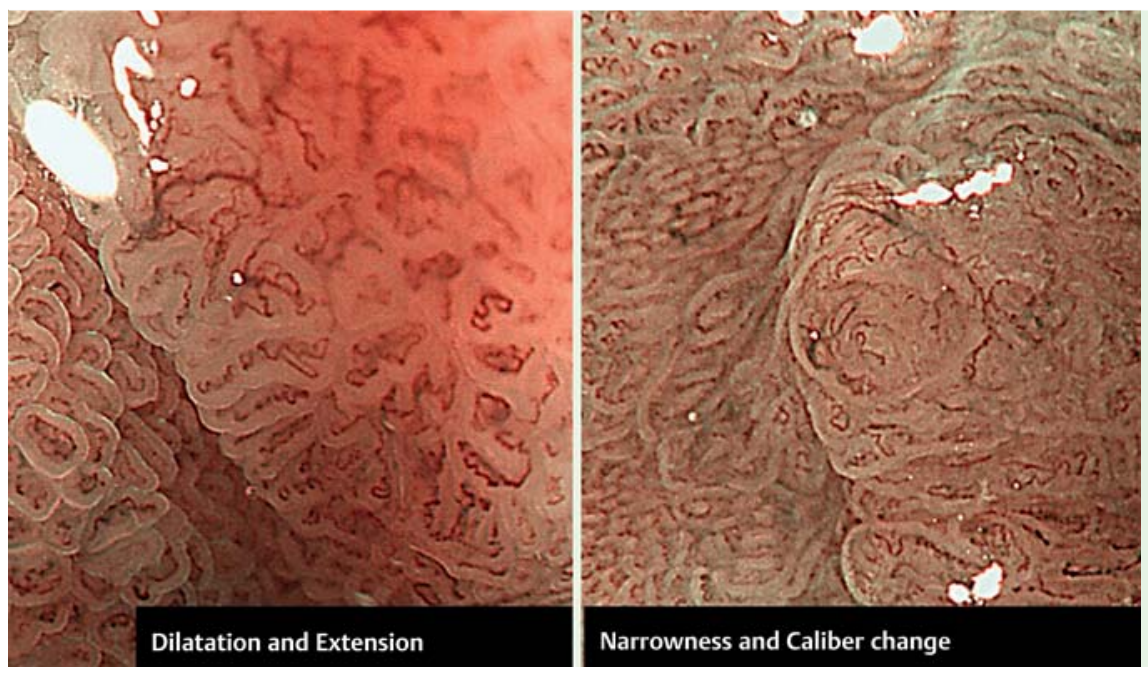

Fig. 2 ME-NBI findings showing the MV specifications.

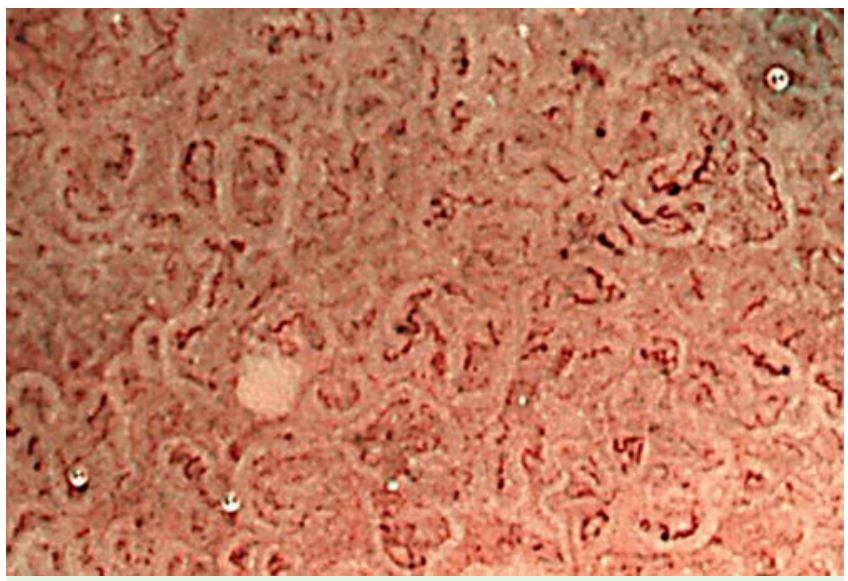

Fig. 3 ME-NBI findings showing an area with MV irregularity.
We also investigated the relationship between the post ESD histological diagnoses (EC or LGA) and the aforementioned clinical and endoscopic characteristics.

\section{Efficacy of each diagnostic modality for discriminating between EC and LGA}

We conducted the following investigation to clarify the usefulness of ME-NBI findings for discriminating EC. We set up three diagnostic criteria for discriminating between EC and LGA in the superficial elevated-type gastric epithelial neoplasias as follows.

1. Preoperative biopsy criterion: all material in the present study had been diagnosed as EC or LGA by biopsy before ESD.

2. CE-WLI criterion: we defined "a lesion greater than $20 \mathrm{~mm}$ in diameter or a reddish-colored lesion" as EC when using conventional endoscopy with white light (CE-WLI) based on our previous research for differential diagnosis using CE-WLI in gastric superficial elevated epithelial neoplasias [16].

3. Vessel plus surface (VS) classification criterion: we defined lesions as EC according to the diagnostic criterion proposed by 

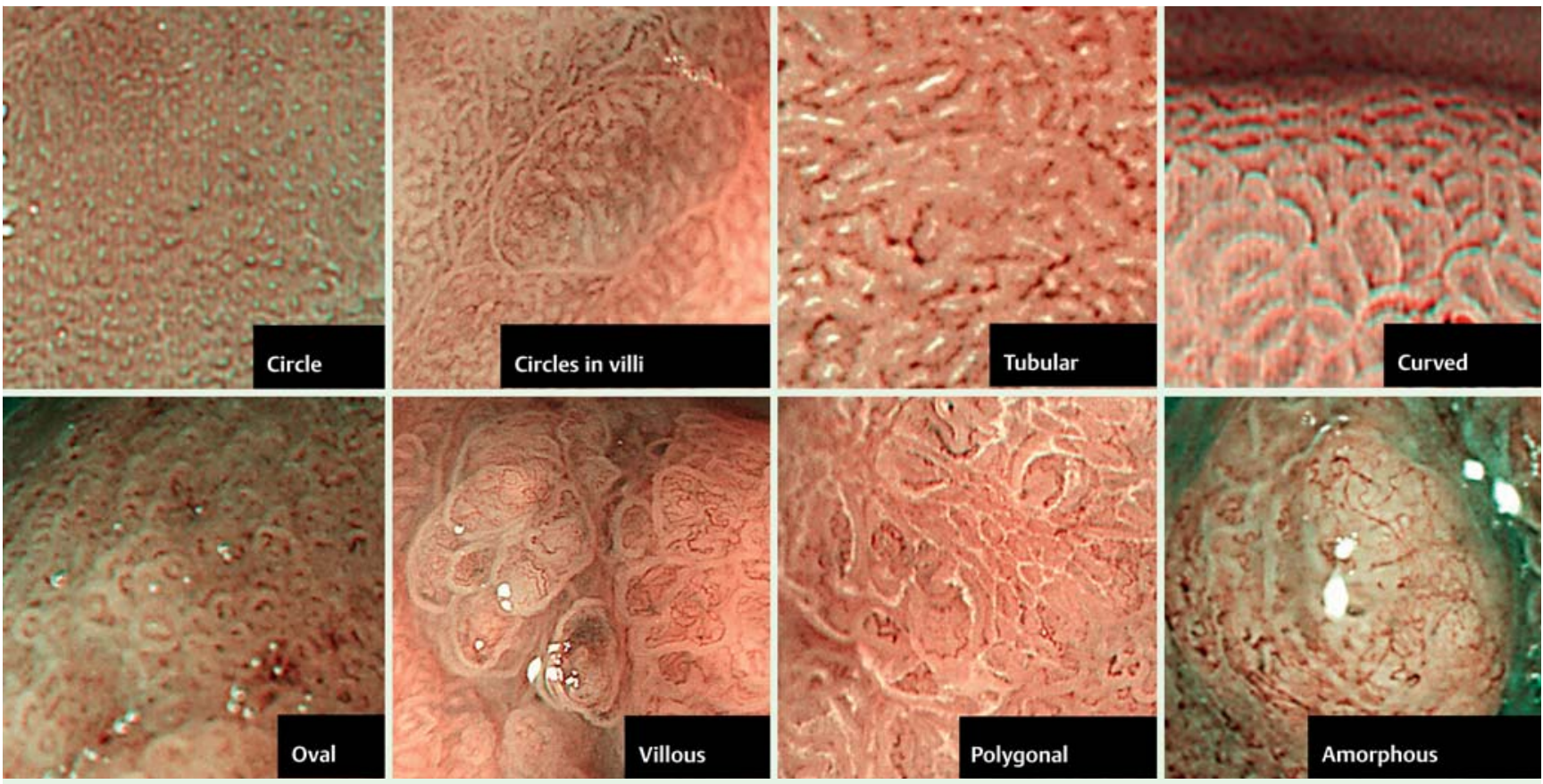

Fig. 4 Eight patterns of the microsurface structure (MS) shape using ME-NBI.
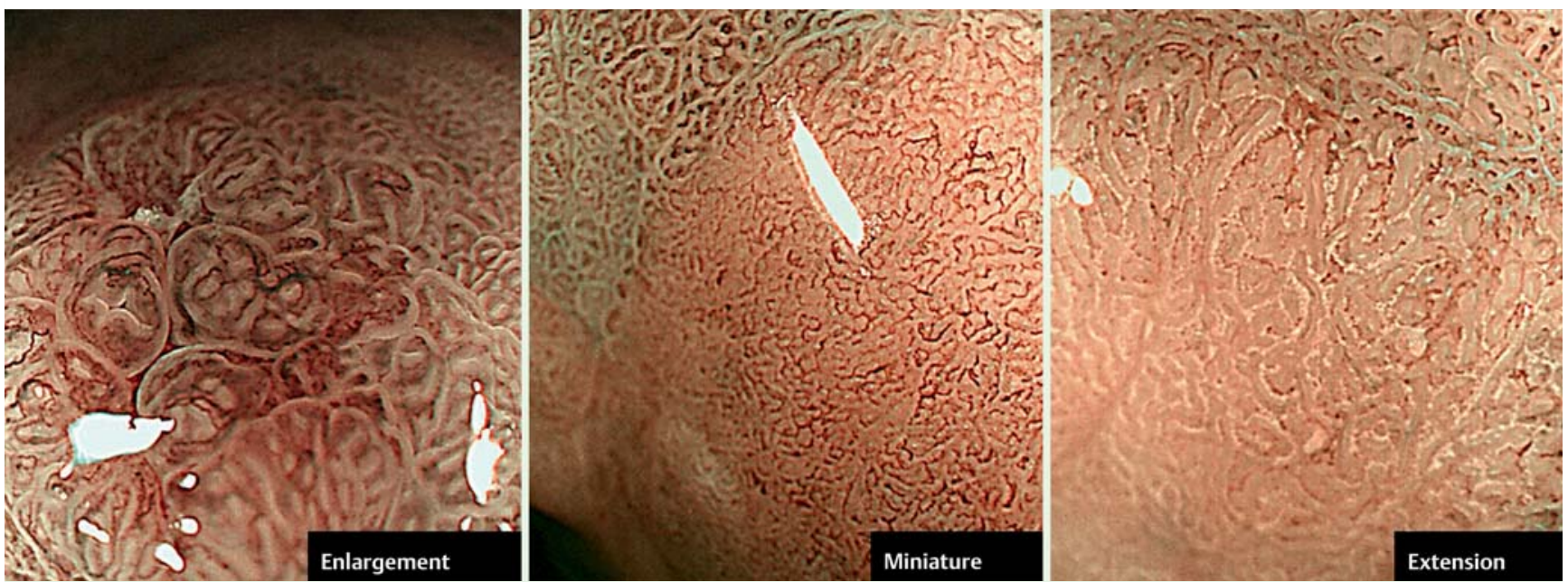

Fig. 5 ME-NBI findings showing the MS specifications.

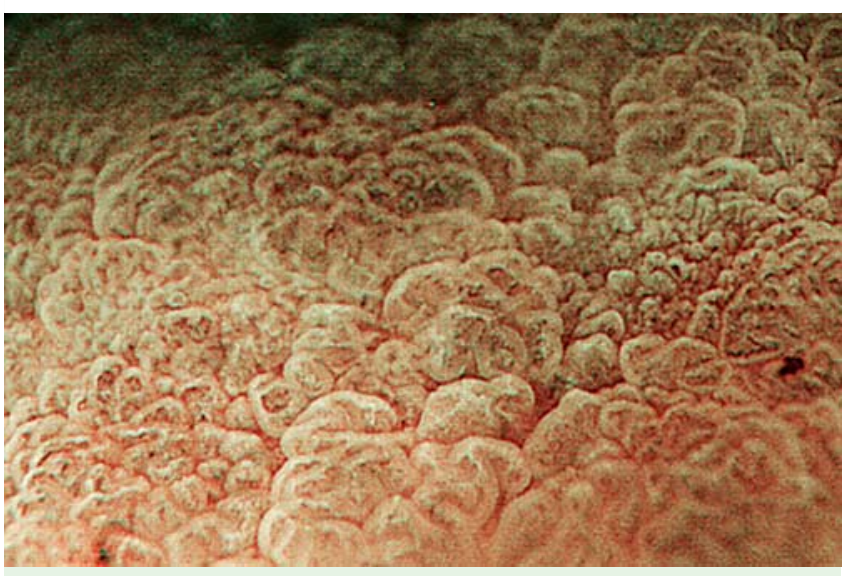

Fig. 6 ME-NBI findings showing an area with MS irregularity.
Yao et al. [7], in which ME-NBI findings of EC include "the presence of an irregular MV pattern with a demarcation line (DL), the presence of an irregular MS pattern with a DL, or both".

Additionally, the sensitivity, specificity, positive predictive value, negative predictive value, likelihood ratio of a positive test, likelihood ratio of a negative test, and diagnostic accuracy for the identification of EC were calculated for each diagnostic criterion.

\section{Statistical analysis}

We used Fisher's exact test for categorical comparison of the data. Differences for continuous data were compared using the MannWhitney $U$ test. The level of significance was set at $P<0.05$. All of the statistical analyses were performed using IBM SPSS Statistics 22 (IBM Co., Chicago, Illinois, United States) and EZR (Saitama Medical Center, Jichi Medical University, Japan) [17]. 
Table 2 Univariate analysis of the clinical characteristics and the conventional endoscopy with white light (CE-WLI) findings in cases with superficially elevated lesions diagnosed as EC and LGA $(n=100)$.

\begin{tabular}{|lclc|} 
& EC $(\mathbf{n = 7 9 )}$ & LGA $(\mathbf{n = 2 1 )}$ & $\boldsymbol{P}$ value \\
\hline Age, median, range, years & $75,61-85$ & $74,63-85$ & $0.560^{1}$ \\
\hline Sex, men/women & $64 / 15$ & $20 / 1$ & $0.181^{2}$ \\
\hline Lesion site, $\mathrm{U} / \mathrm{M} / \mathrm{L}$ & $8 / 35 / 36$ & $1 / 11 / 9$ & $0.817^{2}$ \\
\hline Size, median, range, $\mathrm{mm}$ & $14,2-95$ & $5,2-16$ & $<0.001^{1}$ \\
\hline Diameter, $<20 \mathrm{~mm} / \geq 20 \mathrm{~mm}$ & $58 / 21$ & $21 / 0$ & $0.005^{2}$ \\
\hline Color, reddish/whitish & $48 / 31$ & $9 / 12$ & $0.214^{2}$
\end{tabular}

EC, early carcinoma; LGA, low grade adenoma; $\mathrm{U}$, upper part of the stomach;

$\mathrm{M}$, middle part of the stomach; L, lower part of the stomach.

${ }^{1}$ Mann-Whitney $\mathrm{U}$ test

${ }^{2}$ Fisher's exact test.

Table 3 Univariate analysis of the microvascular architecture (MV) shapes observed using magnifying endoscopy with narrow-band imaging (ME-NBI) in cases with superficially elevated lesions diagnosed as EC or LGA $(n=100)$.

\begin{tabular}{|llcl|}
\hline & EC (n= 79) & LGA (n=21) & P value \\
\hline Closed loop: absent/present & $39 / 40$ & $9 / 12$ & $0.631^{1}$ \\
\hline Open loop: absent/present & $42 / 37$ & $10 / 11$ & $0.807^{1}$ \\
\hline Kinked: absent/present & $17 / 62$ & $6 / 15$ & $0.562^{1}$ \\
\hline Linear: absent/present & $59 / 20$ & $15 / 6$ & $0.783^{1}$ \\
\hline Wavy: absent/present & $18 / 61$ & $10 / 11$ & $0.031^{1}$ \\
\hline Branched: absent/present & $73 / 6$ & $21 / 0$ & $0.338^{1}$ \\
\hline Coiled: absent/present & $74 / 5$ & $20 / 1$ & $1.000^{1}$ \\
\hline Dot: absent/present & $45 / 34$ & $12 / 9$ & $1.000^{1}$ \\
\hline
\end{tabular}

EC, early carcinoma; LGA, low grade adenoma.

${ }^{1}$ Fisher's exact test.

Table 4 Univariate analysis of the MV specifications and areas with MV irregularity observed using ME-NBI in patients with superficially elevated lesions diagnosed as EC or LGA $(n=100)$.

\begin{tabular}{|llll|}
\hline & EC $(\mathbf{n = 7 9 )}$ & LGA (n=21) & $\boldsymbol{P}$ value \\
\hline Dilatation: absent/present & $18 / 61$ & $9 / 12$ & $0.095^{1}$ \\
\hline Narrowness: absent/present & $70 / 9$ & $19 / 2$ & $1.000^{1}$ \\
\hline Extension: absent/present & $21 / 58$ & $11 / 10$ & $0.035^{1}$ \\
\hline Caliber change: absent/present & $49 / 30$ & $18 / 3$ & $0.065^{1}$ \\
\hline MV irregularity: absent/present & $13 / 66$ & $11 / 10$ & $0.001^{1}$
\end{tabular}

EC, early carcinoma; LGA, low grade adenoma; MV, microvascular architecture. ${ }^{1}$ Fisher's exact test.

\section{Ethics}

The present study, under clinical trial registry number UMIN000012925, was conducted in accordance with the Declaration of Helsinki. The institutional review board of Yokohama City University Hospital approved the study protocol. Informed consent was obtained from all participants not only for the endoscopic treatment but also for the use of the patients' clinical data for research purposes.

\section{Results}

\section{$\nabla$}

Among 100 epithelial neoplasias from 91 patients, 21 were diagnosed as LGA (category 3 of the revised Vienna classification) after ESD. Additionally, 79 lesions were diagnosed as EC, and of these, 77 displayed mucosal high grade neoplasias (27 category $4.1,40$ category $4.2,3$ category 4.3 , and 7 category 4.4 ), and 2 displayed submucosal invasive neoplasias (category 5).
Table 5 Univariate analysis of the microsurface structure (MS) shapes observed using ME-NBI in patients with superficially elevated lesions diagnosed as EC or LGA $(n=100)$.

\begin{tabular}{|llcl|}
\hline & EC (n= 79) & LGA (n=21) & P value \\
\hline Circle: absent/present & $31 / 48$ & $8 / 13$ & $1.000^{1}$ \\
\hline Circles in villi: absent/present & $72 / 7$ & $18 / 3$ & $0.434^{1}$ \\
\hline Tubular: absent/present & $16 / 63$ & $1 / 20$ & $0.113^{1}$ \\
\hline Curved: absent/present & $61 / 18$ & $19 / 2$ & $0.230^{1}$ \\
\hline Oval: absent/present & $55 / 24$ & $15 / 6$ & $1.000^{1}$ \\
\hline Villous: absent/present & $33 / 46$ & $15 / 6$ & $0.026^{1}$ \\
\hline Polygonal: absent/present & $52 / 27$ & $18 / 3$ & $0.108^{1}$ \\
\hline Amorphous: absent/present & $60 / 19$ & $19 / 2$ & $0.228^{1}$ \\
\hline
\end{tabular}

EC, early carcinoma; LGA, low grade adenoma.

${ }^{1}$ Fisher's exact test.

Table 6 Univariate analysis of the MS specifications and areas with MS irregularity observed using ME-NBI in patients with superficially elevated lesions diagnosed as EC or LGA $(n=100)$.

\begin{tabular}{|llll|}
\hline & EC $(\mathbf{n = 7 9 )}$ & LGA (n=21) & P value \\
\hline Enlargement: absent/present & $44 / 35$ & $17 / 4$ & $0.044^{1}$ \\
\hline Miniature: absent/present & $31 / 48$ & $8 / 13$ & $1.000^{1}$ \\
\hline Extension: absent/present & $63 / 16$ & $20 / 1$ & $0.113^{1}$ \\
\hline MS irregularity: absent/present & $25 / 54$ & $13 / 8$ & $0.021^{1}$
\end{tabular}

EC, early carcinoma; LGA, low grade adenoma; MS, microsurface structure.

${ }^{1}$ Fisher's exact test.

- Table 2 shows comparisons of the clinical characteristics and the CE-WLI findings between the patients with EC and LGA. The results of the univariate analysis showed that the maximal lesion diameter was significantly greater in EC than in LGA cases $(P<$ $0.001)$. When lesions were divided into a group with a diameter greater than or equal to $20 \mathrm{~mm}$ and a group with a diameter less than $20 \mathrm{~mm}$, there were also significant differences between EC and LGA $(P=0.005)$.

- Table 3 shows comparisons of the MV shapes observed using ME-NBI between EC and LGA. As for the MV shapes, the frequency of a wavy form was a significant predictive factor for EC $(P=$ $0.031)$.

- Table 4 shows the results of the univariate analysis of the MV specifications and the area of MV irregularity when using MENBI to compare EC and LGA. From these results, the frequency of extension in the MV specifications and the area of MV irregularity were significant predictive factors for EC $(P=0.035$ and $P=$ 0.001 , respectively).

- Table 5 shows a comparison of the MS shapes observed using ME-NBI between EC and LGA. As for the MS shapes, the frequency of a villous form was a significant predictive factor for EC ( $P=$ $0.026)$.

- Table 6 shows the results of the univariate analysis of MS specifications and the area of MS irregularity using ME-NBI to compare EC and LGA. From these results, the frequency of enlargement in the MS specifications and the area of MS irregularity were significant predictive factors for EC $(P=0.044$ and $P=0.021$, respectively).

\section{Additional study}

According to the study results described above, we proposed "complex pattern criterion" for discriminating EC using ME-NBI. Namely, we defined lesions as EC, in which ME-NBI findings of EC included "the presence of a wavy form in the MV shapes, the 


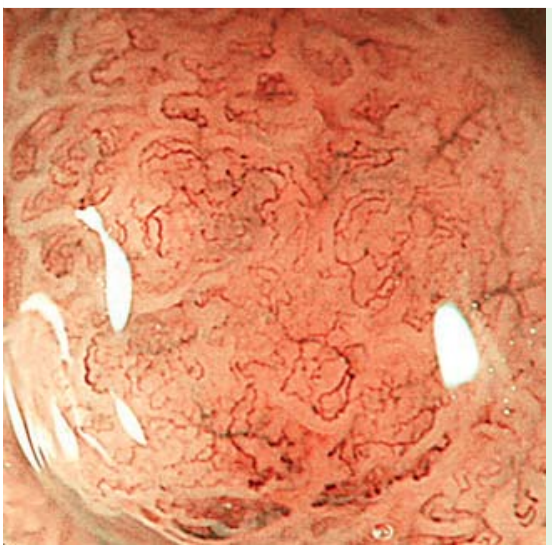

Fig.7 ME-NBI findings showing a complex pattern.

presence of a villous form in the MS shapes, or the presence of an area with MV/MS irregularity" ( $\bullet$ Fig. 7). In the cases where MENBI findings varied partially in a lesion, we diagnosed the lesion as EC if even a part of a positive finding of the complex pattern criterion was present.

- Table 7 shows the diagnostic efficacy of each of the diagnostic modalities for discriminating between EC and LGA, including the preoperative biopsy criterion, CE-WLI criterion, VS classification criterion, and complex pattern criterion ( $\bullet$ Table 7 ). The diagnostic modalities using ME-NBI, namely the VS classification criterion or complex pattern criterion, showed greater sensitivity and diagnostic accuracy for discriminating EC compared with the other modalities.

\section{Discussion}

\section{$\nabla$}

This study focused on the gross appearance of superficial elevated lesions because of the difficulty in the differential diagnosis between EC and LGA in the clinical setting; this limitation is in contrast to cases of depressed gastric epithelial neoplasias, which are almost always carcinomas. LGA may also present with a depressed appearance, but such cases are relatively rare and their malignant potential is generally considered to be greater than that of the elevated type $[18,19]$. Conversely, gastric superficial elevated epithelial neoplasias include not only cases of LGA but also many cases of EC.

Biopsy is an essential method to differentiate between carcinomas and adenomas; however, we frequently encounter inconsistencies between the histological findings from biopsy specimens and resected specimens. The reason for an imprecise diagnosis from biopsy specimens is that parts of the carcinoma may not be included in the sampling or that small biopsy samples often do not contain enough tissue for the correct identification of malignancy. Additionally, several studies have indicated that the diagnostic precision of biopsy for gastric epithelial neoplasias is insufficient [5,6].

The recent introduction of advanced technologies, such as MENBI, has facilitated visualization of the MV below the mucosal epithelium as well as MS of the mucosal epithelium. Due to the incorporation of these visualized microanatomies, the usefulness of ME-NBI for the discrimination between cancerous and noncancerous lesions in the stomach has frequently been reported [7-9]. However, most of these studies were conducted with flat or depressed lesions, and the usefulness of ME-NBI for superficial elevated lesions has not yet been sufficiently investigated. From experience with ME-NBI in practice, ME-NBI findings of EC cases with flat or depressed gross appearances show both disordered irregularity in the MV and obscurity or apparent irregularity in the MS, whereas those with superficial elevated gross appearances frequently show both a mesh-patterned MV or various shaped MV in a glandular structure and well-bordered shapes in the MS. Therefore, we frequently face the difficulty of distinguishing between EC and non-cancerous lesions (including LGA) in superficial elevated lesions.

Currently, there are several published reports of studies focusing on the differential diagnosis of gastric superficial elevated epithelial neoplasias between EC and LGA using ME-NBI [10 - 13]. Nakamura et al. investigated the differences of incidence of the MENBI findings, which were divided into superficial structures (SSs) and irregular microvascular patterns (IMVPs), between EC and LGA [10]. Nonaka et al. evaluated whether the tumor typing by ME-NBI that they proposed is useful for the differential diagnosis of EC and LGA [11]. These findings are partially similar to our results, however, our ME-NBI findings to predict EC in gastric superficial elevated epithelial neoplasias are what were derived from comparisons of the various MV/MS shapes or specifications

Table 7 Diagnostic efficacy of each of the diagnostic modalities for discriminating between EC and LGA, including the preoperative biopsy criterion, CE-WLI criterion, VS classification criterion and complex pattern criterion.

\begin{tabular}{lllll} 
& Preoperative biopsy & CE-WLI & VS classification & Complex pattern \\
\hline Sensitivity & 0.468 & 0.709 & 0.848 & 0.861 \\
$(95 \% \mathrm{Cl})$ & $(0.353-0.585)$ & $(0.596-0.806)$ & $(0.75-0.919)$ & $(0.765-0.928)$ \\
\hline Specificity & 0.857 & 0.571 & 0.476 & 0.389 \\
$(95 \% \mathrm{Cl})$ & $(0.637-0.97)$ & $(0.34-0.782)$ & $(0.257-0.702)$ & $(0.146-0.57)$ \\
\hline PPV & 0.923 & 0.862 & 0.859 & 0.829 \\
$(95 \% \mathrm{Cl})$ & $(0.791-0.984)$ & $(0.753-0.935)$ & $(0.762-0.927)$ & $(0.73-0.903)$ \\
\hline NPV & 0.305 & 0.343 & 0.455 & 0.389 \\
$(95 \% \mathrm{Cl})$ & $(0.192-0.439)$ & $(0.191-0.522)$ & $(0.244-0.678)$ & $(0.173-0.643)$ \\
\hline LR+ & 3.273 & 1.654 & 1.619 & 1.291 \\
$(95 \% \mathrm{Cl})$ & $(1.118-9.584)$ & $(0.99-2.765)$ & $(1.066-2.46)$ & $(0.942-1.769)$ \\
\hline LR & 0.621 & 0.509 & 0.319 & 0.418 \\
$(95 \% \mathrm{Cl})$ & $(0.473-0.816)$ & $(0.307-0.845)$ & $(0.16-0.634)$ & $(0.185-0.945)$ \\
\hline Diagnostic accuracy & 0.551 & 0.68 & 0.77 & 0.75 \\
$(95 \% \mathrm{Cl})$ & $(0.447-0.652)$ & $(0.579-0.77)$ & $(0.675-0.848)$ & $(0.653-0.831)$ \\
\hline
\end{tabular}

CE-WLI, conventional endoscopy with white light; VS, vessel plus surface; PPV, positive predictive value; NPV, negative predictive value; LR + , likelihood ratio of a positive test; LR - , likelihood ratio of a negative test; $95 \% \mathrm{Cl}, 95 \%$ confidence interval. 
between EC and LGA. Additionally, these data cannot easily be compared to the findings in the present study because some of these earlier studies investigated gastric epithelial neoplasias that had been diagnosed as LGA based on preoperative biopsies but were identified as EC or LGA after ESD, occasionally without strict distinction of the macroscopic appearance of elevation or depression. Meanwhile, in the present study, 100 consecutive cases of gastric superficial elevated epithelial neoplasias (79 EC and 21 LGA) were evaluated retrospectively to clarify the characteristics of the ME-NBI findings for discriminating EC from LGA, regardless of whether the preoperative diagnosis was EC or LGA. The present study revealed that wavy MV shapes, extensions in MV specifications, villous MS shapes, enlargement in MS specifications, and areas with MV or MS irregularity were useful MENBI findings for discriminating between EC and LGA in gastric superficial elevated lesions. We defined a complex pattern for the identification of EC as follows. ME-NBI findings of EC included the presence of a wavy form in the shape of the MV, the presence of a villous form in the shape of the MS, or the presence of an area with MV/MS irregularity. As a result, MV specifications required an extension when the MV shapes showed a wavy form, and MS specifications required an enlargement when the MS shape showed a villous form.

We also investigated why MV/MS irregularities on ME-NBI findings were more frequently observed in EC than LGA. When considering the histological structure, the epithelial glands usually have a narrow width and are arranged in an orderly fashion in LGA, whereas these glands are quite often formed non-uniformly and are arranged in a disorderly manner in EC. In addition, angiogenesis is a well-known and important factor in gastrointestinal carcinogenesis and tumor progression [20,21]. From these findings, differences in the histological structure and angiogenesis between EC and LGA may produce different ME-NBI findings when the surface of a lesion is observed vertically.

Yao et al. proposed a diagnostic system called the VS classification system for differentiating between cancerous and noncancerous lesions, in which the ME-NBI findings of EC include the presence of an irregular MV pattern with a demarcation line (DL), the presence of an irregular MS pattern with a DL, or both [7]. Based on this VS classification, several studies have reported good outcomes for the differential diagnosis of gastric lesions [9, 22,23]. In research similar to our study, Maki et al. retrospectively analyzed the ME-NBI findings of 93 gastric superficial elevated lesions (61 EC and 32 LGA) resected by endoscopic resection, and these authors reported that the sensitivity, specificity, and accuracy of endoscopic diagnosis using VS classification was $95 \%, 88 \%$, and $92 \%$, respectively [24].

Conversely, these measures using endoscopic diagnosis and the complex pattern criterion based on our results were $86.1 \%$, $38.9 \%$, and $75 \%$, respectively. In comparison, using the VS classification system, these measures were $84.8 \%, 47.6 \%$, and $77 \%$, respectively, in the present study. The reason for this difference in diagnostic efficacy between the previous report and the present study remains unclear. However, we speculate that the discrepancies in these results are likely due to the retrospective nature of our study. There are several limitations to this study. The main limitation was that it was a cross-sectional retrospective study, and the endoscopic images that were reviewed retrospectively might be insufficient for the qualitative diagnosis of gastric superficial elevated neoplasias. Regarding the judgment for EC, when we encountered a lesion having different ME-NBI findings in each of its parts, we diagnosed the lesion as EC even if a part of a positive finding of the complex pattern criterion was present. The exact number of lesions having both positive and negative findings of complex pattern criterion within the same lesion was unknown in the present study, because this study was a retrospective analysis. This may have affected the results of the present study. Additionally, our sample size was relatively small, and the number of LGA cases was only 21 . Therefore, retrospective analysis of the prevalence of EC in the present study could account for the difference in the diagnostic efficacy between the previous report and the present study. In the future, further investigation should be conducted in an adequate study design. Despite these limitations, the present study provided the characteristics of MENBI findings for EC with a superficial elevated gross appearance, and suggested the usefulness of ME-NBI for the differential diagnosis of EC and LGA in gastric superficial elevated epithelial neoplasias.

\section{Competing interests: None}

\section{Institutions}

${ }^{1}$ Department of Gastroenterology and Hepatology, Yokohama City University School of Medicine, Yokohama, Japan

${ }^{2}$ Department of Medical Education, Yokohama City University School of Medicine, Yokohama, Japan

${ }^{3}$ Department of Gastroenterology, Hiratsuka City Hospital, Hiratsuka, Kanagawa, Japan

${ }^{4}$ Department of Pathology, Yokohama City University Graduate School of Medicine, Yokohama, Kanagawa, Japan

\section{Acknowledgments}

$\nabla$

This work was supported by the Yokohama Foundation for the Advancement of Medical Science.

\section{References}

1 Japanese Gastric Cancer Association. Japanese classification of gastric carcinoma: 3rd English edition. Gastric Cancer 2011; 14: 101-112

2 Japanese Gastric Cancer Association. Japanese gastric cancer treatment guidelines 2010 (ver. 3). Gastric Cancer 2011; 14: 113-123

3 Rugge M, Farinati F, Baffa R et al. Gastric epithelial dysplasia in the natural history of gastric cancer: a multicenter prospective follow-up study. Interdisciplinary Group on Gastric Epithelial Dysplasia. Gastroenterology 1994; 107: 1288-1296

4 Yamada H, Ikegami M, Shimoda T et al. Long-term follow-up study of gastric adenoma/dysplasia. Endoscopy 2004; 36: 390 - 396

5 Yoon WJ, Lee DH, Jung YJ et al. Histologic characteristics of gastric polyps in Korea: emphasis on discrepancy between endoscopic forceps biopsy and endoscopic mucosal resection specimen. World J Gastroenterol 2006; 12: 4029-4032

6 Kim YJ, Park JC, Kim JH et al. Histologic diagnosis based on forceps biopsy is not adequate for determining endoscopic treatment of gastric adenomatous lesions. Endoscopy 2010; 42: 620-626

7 Yao K, Anagnostopoulos GK, Ragunath K. Magnifying endoscopy for diagnosing and delineating early gastric cancer. Endoscopy 2009; 41: $462-467$

8 Kato M, Kaise M, Yonezawa J et al. Magnifying endoscopy with narrowband imaging achieves superior accuracy in the differential diagnosis of superficial gastric lesions identified with white-light endoscopy: a prospective study. Gastrointest Endosc 2010; 72: 523 - 529

9 Ezoe $Y$, Muto M, Uedo $N$ et al. Magnifying narrowband imaging is more accurate than conventional white-light imaging in diagnosis of gastric mucosal cancer. Gastroenterology 2011; 141: 2017-2025

10 Nakamura $M$, Shibata $T$, Tahara $T$ et al. The usefulness of magnifying endoscopy with narrow-band imaging to distinguish carcinoma in flat elevated lesions in the stomach diagnosed as adenoma by using biopsy samples. Gastrointest Endosc 2010; 141: 1070-1075

11 Nonaka K, Arai S, Ban S et al. Prospective study of the evaluation of the usefulness of tumor typing by narrow band imaging for the differential diagnosis of gastric adenoma and well-differentiated adenocarcinoma. Dig Endosc 2011; 23: 146-152 
12 Miwa K, Doyama $H$, Ito $R$ et al. Can magnifying endoscopy with narrow band imaging be useful for low grade adenomas in preoperative biopsy specimens? Gastric Cancer 2012; 15: 170-178

13 Tsuji Y, Ohata K, Sekiguchi M et al. Magnifying endoscopy with narrowband imaging helps determine the management of gastric adenomas. Gastric Cancer 2012; 15: 414-418

14 Gotoda T, Yanagisawa A, Sasako $M$ et al. Incidence of lymph node metastasis from early gastric cancer: estimation with a large number of cases at two large centers. Gastric Cancer 2000; 3: 219-225

15 Dixon MF. Gastrointestinal epithelial neoplasia: Vienna revisited. Gut 2002; 51: $130-131$

16 Nonaka T, Inamori M, Kanoshima K et al. Evaluation of endoscopic findings for discriminating between early carcinomas and low-grade adenomas in superficial elevated gastric lesions. Turk J Gastroenterol 2016; 27: 108 - 114

17 Kanda $Y$. Investigation of the freely available easy-to-use software 'EZR' for medical statistics. Bone Marrow Transpl 2013; 48: 452 - 458

18 Xuan ZX, Ambe K, Enjoji M. Depressed adenoma of the stomach, revisited. Histologic, histochemical, and immunohistochemical profiles. Cancer 1991; 67: 2382-2389
19 Tamai N, Kaise M, Nakayoshi T et al. Clinical and endoscopic characterization of depressed gastric adenoma. Endoscopy 2006; 38: 391 - 394

20 Folkman J, Klagsbrun M. Angiogenic factors. Science 1987; 235: 442 447

$21 \mathrm{Liu} H, \mathrm{Li} Y \mathrm{Y}, \mathrm{Yu}$ T et al. Confocal endomicroscopy for in vivo detection of microvascular architecture in normal and malignant lesions of upper gastrointestinal tract. J Gastroenterol Hepatol 2008; 23: 56-61

22 Tao G, Xing-Hua L, Ai-Ming Y et al. Enhanced magnifying endoscopy for differential diagnosis of superficial gastric lesions identified with white-light endoscopy. Gastric Cancer 2014; 17: 122 -129

23 Yao K, Doyama H, Gotoda $T$ et al. Diagnostic performance and limitations of magnifying narrow-band imaging in screening endoscopy of early gastric cancer: a prospective multicenter feasibility study. Gastric Cancer 2014; 17: 669-679

24 Maki S, Yao K, Nagahama T et al. Magnifying endoscopy with narrowband imaging is useful in the differential diagnosis between low-grade adenoma and early cancer of superficial elevated gastric lesions. Gastric Cancer 2013; 16: 140-146 